\title{
Microtitre plate measurement of platelet response to hypotonic stress
}

\author{
A FARRUGiA, C HUGHES, S DOUGLAS, M NEAL, J JAMES Blood Processing and \\ Haematology Departments, Red Cross Blood Bank, South Melbourne, Victoria, Australia
}

SUMMARY The conventional method of assessing the platelet response to hypotonic stress (HSR) was adapted to allow microtitre plate technology to be used. After water is added to a platelet suspension two sequential readings are taken at $414 \mathrm{nM}$ on a vertical microplate reader. The difference between the second (three minutes) and the first (one minute) was defined as the HSR. This method allowed the relation between platelet concentrate $\mathrm{pH}$ and viability to be confirmed, and an HSR value for use in quality control was established. The method correlated well with the conventional technique and permitted measurement of undiluted samples as well as of products with a high free haemoglobin concentration.

After exposure to hypotonic stress platelets swell and then contract to their normal shape. This recovery phase requires the integrity of the platelet's biochemical functions and has been related to platelet viability as the hypotonic shock response (HSR). ${ }^{\prime}$

Many blood banks use the test as a measure of the quality of platelet concentrate, but the conventional spectrophotometers used to monitor the response are not part of standard blood bank equipment. Furthermore, the test is rather lengthy to perform and its use in testing large numbers of samples is thus limited. Microtitre plate tests are fast becoming part of standard blood bank methodology used in red cell grouping and virological screening. We therefore attempted to adapt our standard method for performing the platelet HSR to a microtitre plate format, in order to be able to a test a large number of samples on a vertical microplate reader.

\section{Material and methods}

Platelet concentrates were prepared in the components laboratory of the blood processing department of the Red Cross Blood Bank, using standard methods. Briefly, whole blood donations collected in CPD anticoagulant were centrifuged at $1375 \times g$ for five and a half minutes and the platelet-rich plasma was siphoned into a satellite pack. An additive solution $(100 \mathrm{ml})$ was added to the red cell pack which was then sealed. The empty additive pack then served as a container for platelet-poor plasma generated by centrifuging the platelet-rich plasma at $4000 \times g$ for 12 minutes. The sedimented platelets were allowed to rest

Accepted for publication 12 June 1989 for 90 minutes without agitation and were then resuspended on a horizontal agitator. Concentrates were resuspended in about $60 \mathrm{ml}$ of plasma at a platelet concentration of $4-16 \times 100^{11} / 1$. Platelet concentrates were stored with continuous agitation for five days at a controlled temperature of $-20-24^{\circ} \mathrm{C}$.

To prevent contamination samples were taken from $\varnothing$ concentrates by stripping down integral protions of plastic tubing into the pack, allowing platelet suspen-o sion to fill the tubing and sealing off a segment using a heat sealer.

\section{HYPOTONIC SHOCK RESPONSE}

Conventional spectrophotometric method

A Philips SP8 ultraviolet visual spectrophotometer (Pye Unicam Ltd, Cambridge) with an integral chart recorder was used to monitor the response. Hypotonic stress was induced by adding $500 \mathrm{ul}$ of distilled water to $500 \mathrm{ul}$ of platelet suspension. The latter consisted of a 1/4 dilution of platelet concentrate in platelet-poor plasma (platelet count $\left.<5 \times 10^{9} / 1\right)$. The platelet suspension was placed in a microcuvette in the spectrophotometer's cell holder with the instrument's lid open-water was then added and the lid was closed. The change in light absorbance at $420 \mathrm{nM}$ was traced by the chart recorder as the familiar pattern of a decrease followed by a gradual restoration; the absorbance two minutes after the minimum absorbance recorded was defined as the HSR.

\section{Microtitre plate method}

Conventional, round-bottomed microtitre plates were used (Nunc Laboratories, Roskilde, Denmark) and a 12 channel 50-250 ul micropipette (Flow Laboratories, Ayrshire, Scotland) for water delivery 


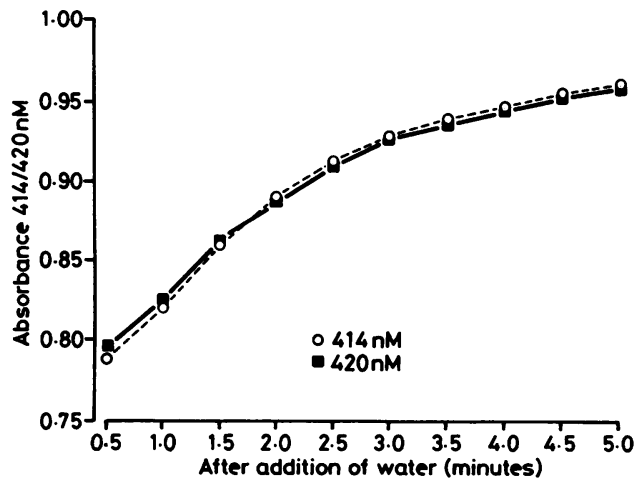

Fig 1 HSR: conventional spectrophotometry

and a Titertek Multiscan MC vertical reader (Flow Laboratories) for recording absorbance changes. After initial development work the test was optimised as follows: 150 ul of platelet concentrate was delivered to each well in a column; in practice two columns of eight wells each could be analysed in one test run. Distilled water ( $150 \mathrm{ul})$ was then delivered to each well by means of the multichannel pipette and a stopwatch was started. One minute after the water had been delivered a reading of the well absorbance was taken on the plate reader at a wavelength of $414 \mathrm{nM}$. A

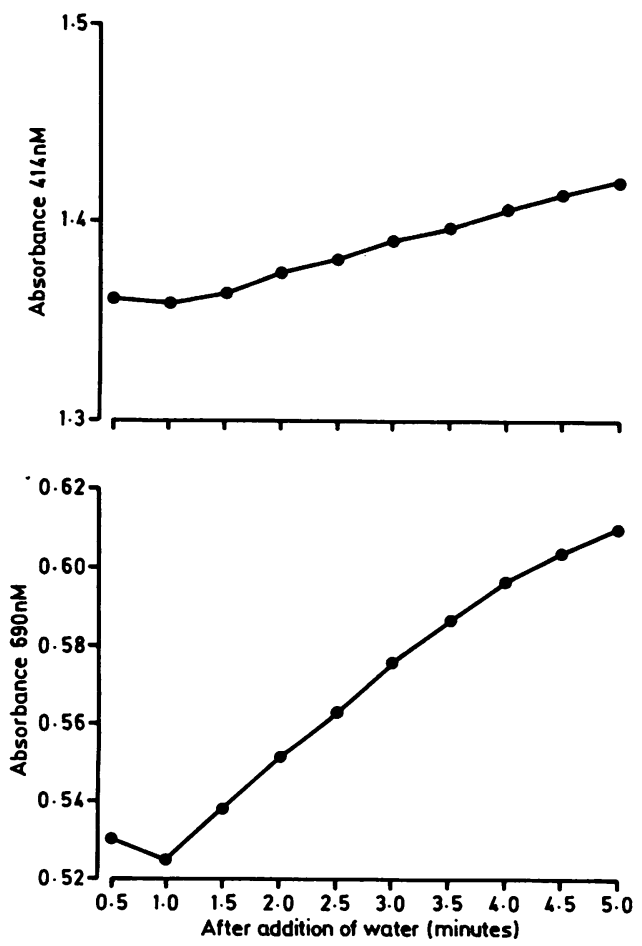

Fig 2 Microtitre HSR: effect of wavelength

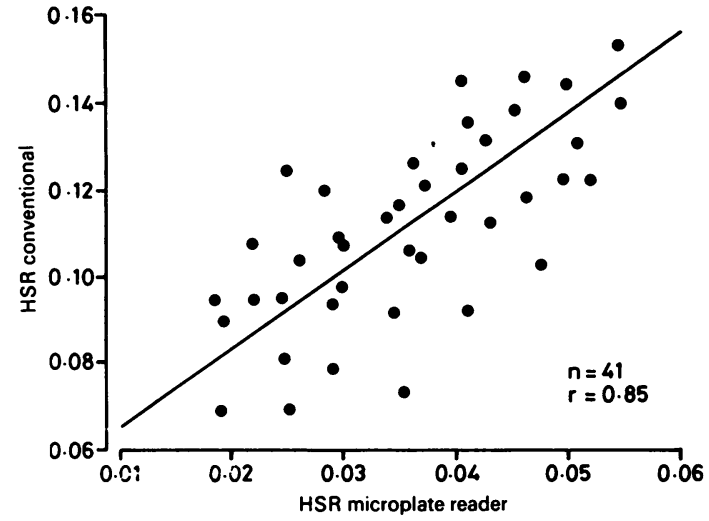

Fig 3 Microtitre HSR: correlation with conventional method (all measurements at $414 \mathrm{nM}$ ).

second reading was also taken three minutes after the water had been delivered. The difference in absorbance between the first and second readings was defined as the HSR.

\section{Results}

Because the microplate reader did not have a $420 \mathrm{nM}$ filter we decided to monitor absorbance changes at 414 nM. Fig 1 shows that equivalent results were obtained at both wavelengths using the Pye Unicam spectrophotometer. The microplate method allowed the response to be monitored at this and at the whole range of wavelengths available on the plate reader (fig 2). Fig 3 shows the correlation obtained using both methods on diluted samples of platelet concentrates. Although dilution is very quickly performed using the multichannel pipette, we now perform tests on neat samples as the results correlate well with those obtained using diluted samples (fig 4).

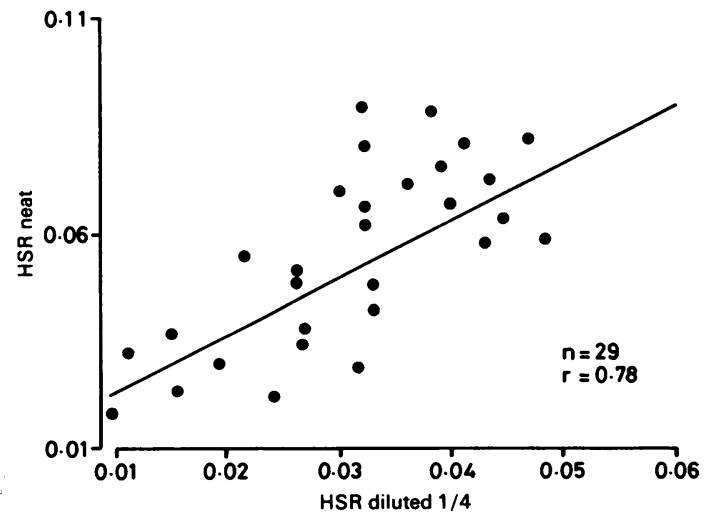

Fig 4 Microtitre HSR: Measurement of neat and diluted samples (all measurements at $414 \mathrm{nM}$ ). 
Table Effect of free haemoglobin on HSR (Mean (SD))*

\begin{tabular}{lll}
\hline & \multicolumn{1}{l}{$H S R$} & \\
\cline { 2 - 3 } Free haemoglobin $(\mathrm{mg} / \mathrm{dl})$ & $414 n M$ & $690 \mathrm{nM}$ \\
\hline 45 & & \\
27 & Out of range & $0.033(0.004)$ \\
22 & $0.032(0.003)$ & $0.036(0.004)$ \\
15 & $0.039(0.002)$ & $0.033(0.003)$ \\
9 & $0.035(0.003)$ & $0.031(0.001)$ \\
\hline
\end{tabular}

*Four determinations at each free haemoglobin concentration.

The table shows that HSR measurements are possible on samples with high free haemoglobin concentrations as are occasionally found on stored blood samples and in red cell contaminated platelet concentrates after hypotonic shock. These results were obtained by diluting fresh platelet concentrate suspensions with different amounts of a haemoglobin-rich plasma prepared by adding a red cell haemolysate to plasma. At the highest concentration studied, the absorbance level was out of the plate reader's range at $414 \mathrm{nM}$. Monitoring at $690 \mathrm{nM}$, however, allowed the HSR to be measured on this sample. HSR values were not affected by the free haemoglobin concentration.

Figs $5 \mathrm{a}$ and $\mathrm{b}$ shows the relation between the HSR and the $\mathrm{pH}$ at expiry with the preservation of the HSR during storage. $\mathrm{pH}$ values below 6.5 show deteriora-
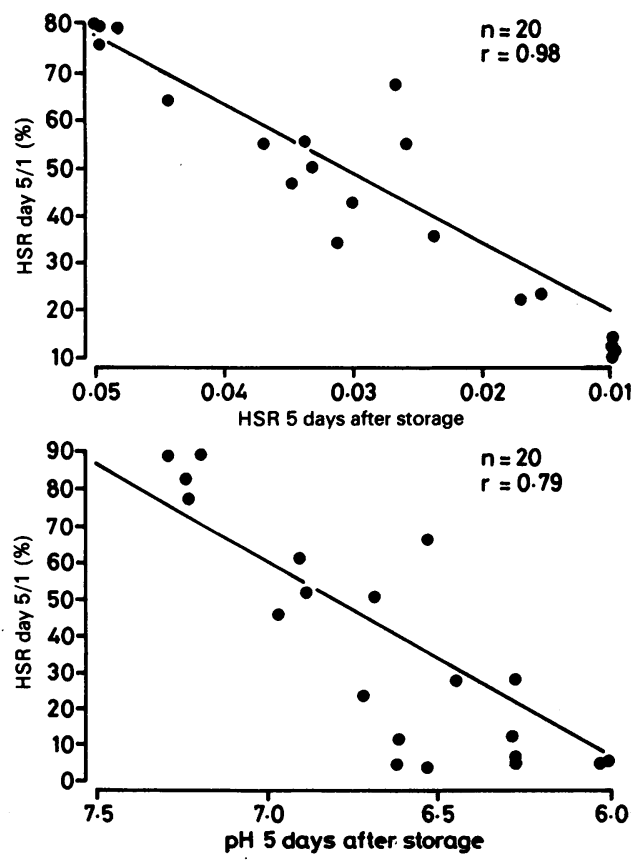

Fig 5 Microtitre HSR: (414 nM): relation between $p H$ and $H S R$ at expiry with preservation of $H S R$. tion of the HSR, with over $60 \%$ of the initial response being lost. These values correspond to absolute HSR values after five days of storage of about 0.02.

\section{Discussion}

Previous studies have shown that measurement of the platelet response to hypotonic stress correlates well with in vivo behaviour after platelet transfusion. ${ }^{12}$ The simplicity of the HSR makes it an attractive test in quality monitoring of these products by blood banks but the previously described spectrophotometric technique is quite lengthy to perform. This is a drawback as adequate quality monitoring of single donor products such as platelet concentrates requires analysis of large numbers of samples. The availability of microtitre plate readers in most blood banks led us to attempt adapting the HSR to this method, which permits simultaneous measurement of many samples. We are satisfied that we have achieved this aim as the microtitre test correlates well with the established method. The latter gives absolute HSR values which are two to three times higher than those obtained on the microplate reader; differences in sample volume and the optical monitoring system may account for this. The relation between the microplate HSR with $\mathrm{pH}$ at expiry also mirrors that of the conventional method $^{3}$ and allows platelet deterioration when the $\mathrm{pH}^{2}$ falls below 6.5 during storage to be shown (fig $5 \mathrm{~b}$ ). Although present quality control criteria do not delineate a normal range for HSR values, we consider that platelets which show a drop of over $60 \%$ of their initial response during storage would be unsuitable for transfusion. On this basis an HSR above 0.02 after storage is taken as a quality assurance indicator of viability.

At present we prefer to limit a batch of tests to 16 samples as we are not able to deliver water to all wells simultaneously and would thus anticipate some discrepancy between early and late samples in a batch if all the 12 columns in a plate were to be used. Our present testing requirements are not hindered by this batch size but use of a reagent delivery system which would allow water to be added to multiple columns simultaneously should allow all 96 wells to be used in every batch.

Measurement at higher wavelengths such as $690 \mathrm{nM}$ allows tests to be performed on samples containing high haemoglobin concentrations which would not be possible at lower wavelengths, and also permits measurement on neat samples with very high platelet counts. We therefore consider that use of the microtitre plate technology introduces a degree of flexibility and convenience to the performance of this test that is not found using conventional spectrophotometers, and we anticipate that the method 
should make this test more accessible to blood banks engaged in quality control of their products.

We thank Mr Greg Cooper, scientist in charge, Virology Laboratory, Red Cross Blood Bank, for the loan of the microplate reader on which these studies were performed, and Dr Gordon Whyte, director of the Red Cross Blood Bank, for reviewing the manuscript.

\section{References}

$1 \mathrm{Kim}$ BK, Baldini MG. The platelet response to hypotonic shock. Its value as an indicator of platelet viability after storage. Transfusion 1974;14:130-8.
2 Valeri CR. Feingold H, Marchionni LD. The relation between response to hypotonic stress and the $\mathrm{Cr}$ recovery in vivo of preserved platelets. Transfusion 1974;14:331-7.

3 Tandy NP, Taylor MA. Platelet concentrates for transfusion: control of production and storage. Med Lab Sci 1980;37:127-36.

Requests for reprints to: Dr A Farrugia, Development Section, Blood Processing Department, Red Cross Blood Bank, cnr. Balston \& Kavanagh Streets, South Melbourne, Victoria, Australia 3205. 